\title{
A Novel Algorithm for Improving the SINR of D2D Communication in the Cellular Network
}

\author{
Cheng Huan ${ }^{1, *}$, Youhua $\mathrm{Fu}^{1,2}$ and Jin Wang ${ }^{3}$ \\ ${ }^{1}$ The Key Lab of Broadband Wireless Communication and Sensor Network \\ Technology, Ministry of Education, Nanjing University of Posts and \\ Telecommunications, Nanjing, 210003, China \\ ${ }^{2}$ National Mobile Communications Research Laboratory, Southeast University, \\ Nanjing, 210096, China \\ ${ }^{3}$ Nanjing University of Information Science and Technology, \\ Nanjing, 210044, China
}

\begin{abstract}
In this paper, we investigate the interference scenario where multiple Device-to-Device (D2D) pairs reuse the spectrum resources allocated to one cellular user, and propose a novel algorithm for improving the signal-to-interference-plus-noise ratio (SINR) of D2D communication. We call this D2D SINR improvement algorithm as DSIA, and design it from the perspective of precoding and decoding. With the DSIA, each D2D pair involved in the network can obtain the corresponding precoding and decoding vectors which make the SINR of its receiver being maximized. In order to clearly describe the design procedure of the proposed algorithm, we first formulate an optimization problem with multiple variables to maximize the SINR of each D2D receiver, then simplify the objective function through mathematical derivation and seek out the relationship between the optimized variables which can transform the optimization problem to one of solving nonlinear equations, and finally propose the DSIA to work out the nonlinear equations. Numerical results show that the DSIA will enable D2D to achieve significant performance gains in terms of SINR and bit-error rate (BER) compared with the traditional spectrum orthogonal scheme.
\end{abstract}

Keywords: D2D communication, cellular network, SINR, BER

\section{Introduction}

Recently, Device-to-Device (D2D) communication in the cellular network has been considered as a promising technology to improve the spectrum efficiency [1-2], by which the problem of resources shortage will be alleviated. Moreover, D2D can reduce the network loading, increase the cellular coverage, and decrease the battery consumption of users [3-4]. Due to these advantages, D2D is becoming a research hotspot.

To guarantee the performance of D2D communication, one of important issues is to control the interference caused by the spectrum non-orthogonal sharing mode in which D2D communication and cellular communication share the same spectrum resources [2]. For this, a receive mode selection scheme was proposed in [5], an interference limited area control scheme was presented in [6], a power optimization scheme was developed in [7], and various schemes based on the precoding and decoding technique were designed in [8-10]. However, these works focus on the interference scenario where only one D2D pair reuse the resources allocated to the cellular user, and the mutual interference between D2D pairs are not taken into account when the same cellular resources are shared by multiple D2D pairs. Although [11] adopts the asymptotic IA scheme [12-13] to deal with the mutual interference problem of multiple D2D pairs, this research was carried out 
within the D2D system in the spectrum orthogonal sharing mode [2], and therefore the interference to D2D receivers from the cellular user was not being considered.

In this paper, we investigate the interference scenario where multiple D2D pairs reuse the same resources allocated to one cellular user, and hence the mutual interference between D2D pairs and the cellular interference to D2D are both involved. According to this consideration, we try to handle the interference problem from the perspective of precoding and decoding, and based on that, a signal-to-interference-plus-noise ratio (SINR) improvement algorithm called DSIA is proposed for D2D. It should be noted that the usual D2D system is a network consisting of single-antenna users, while employing a relay into this system and using the cooperative communication technology [14], it could form a virtual MIMO system by which an effective channel extension is accomplished, and hence the design of precoding and decoding vectors/matrices can be implemented [15-17]. In order to successfully retransmit signals from multiple users, a green relay which can handle multiple transmissions simultaneously [18] is utilized here, and the Amplify-and-Forward (AF) strategy is adopted on this green relay for its simplicity [19]. Based on the D2D system with the introducing of the relay, the interference control problem is first formulated as an optimization problem to maximize the SINR of each D2D receiver. Then, due to the problem complexity caused by multiple variables, the objective function is simplified through mathematical derivation and thus the optimization problem will transform to one that can be settled easier. Finally, the DSIA is proposed to solve the transformed problem and obtain the optimized precoding and decoding vectors. The design procedure of DSIA has been described briefly in [20], and the complete contents are displayed in this paper. Taken as a whole, by means of the optimization processing of all D2D transceivers in the network, the DSIA will make the SINR of each D2D receiver being maximized which achieves the goal of controlling interference.

In order to verify the performance of the proposed novel algorithm, we execute Monte Carlo simulations for it. Numerical results show that in comparison with the traditional spectrum orthogonal scheme $[2,11]$ and the case with no interference control, the DSIA will enable D2D involved to obtain significant performance gains in terms of SINR and bit-error rate (BER).

The rest of this paper is organized as follows. Section 2 describes the system model and problem formulation. Section 3 presents the proposed D2D SINR improvement algorithm (DSIA) solving the optimization problem formulated in the former section. Section 4 shows the simulation results and the performance evaluation of the proposed algorithm. Section 5 summarizes this paper and draws the conclusion.

Notation: Throughout the rest of this paper, lowercase boldface letters are used for vectors and uppercase boldface ones are used for matrices. For an arbitrary vector or matrix, []$^{\mathrm{T}},[]^{\mathrm{H}}$ and []$^{-1}$ denote the transpose, the conjugate transpose and the inverse, respectively. Besides, $\operatorname{tr}[]$ and $\lambda_{\max }[]$ denote the trace and the largest eigenvalue of any general matrix, and $\mathbf{e}_{\max }[]$ denotes the corresponding eigenvector of $\lambda_{\max }[]$.

\section{System Model and Problem Formulation}

We consider a single cell interference scenario as in Figure 1. There exist one base station, one cellular user indicated by $\mathrm{C}$, one green $\mathrm{AF}$ relay indicated by $\mathrm{R}$, and $M$ D2D pairs involving the transmitter $i$ indicated by $\mathrm{S}_{i}$ with its corresponding receiver $i$ indicated by $\mathrm{D}_{i}$, where $i \in \Omega$ and $\Omega=\{1, \ldots, M\}$. In this scenario, each user is assumed to be equipped with a single antenna, and D2D pairs are assumed to reuse the same spectrum resources allocated to the cellular user. In particular, uplink resource sharing is taken into account due to its under-utilization [3], and therefore the cellular interference to D2D receivers and the mutual interference between multiple D2D pairs will produce in 
the process of D2D communication.

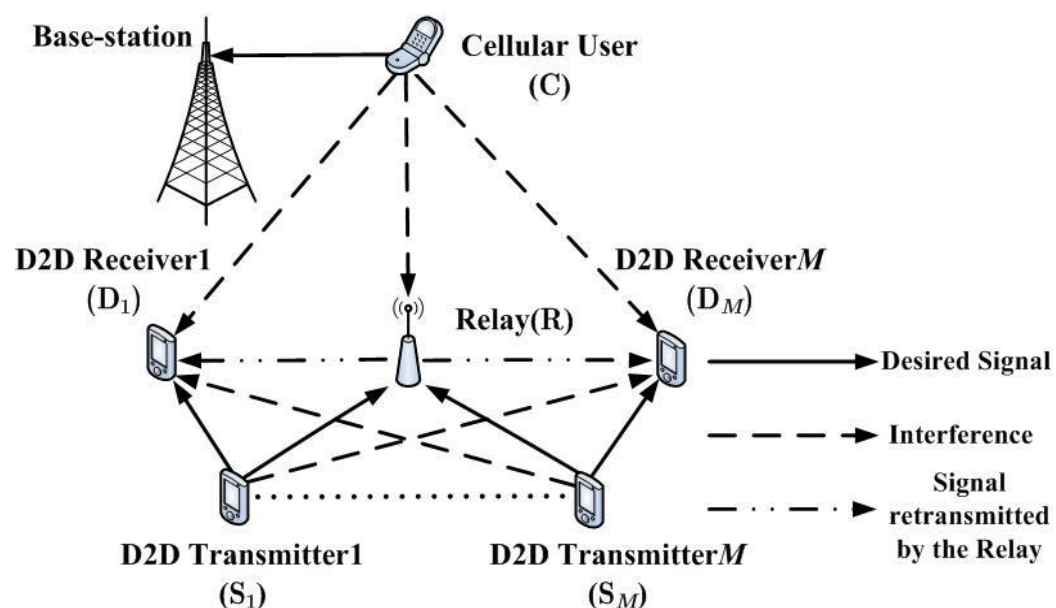

Figure 1. System Model of D2D Communication in the Cellular Network with the Introducing of a Green AF Relay

According to the description of system model, an optimization problem to maximize the SINR of each D2D receiver will be described in the remainder of this section.

First, the received signal at each $\mathrm{D} 2 \mathrm{D}$ receiver (i.e., $\mathrm{D}_{i}, i \in \Omega$ ) through the direct link in time-slot 1 is written as

$$
y_{\mathrm{D}_{i}}^{(1)}=h_{\mathrm{S}_{i} \mathrm{D}_{i}}^{(1)} x_{\mathrm{S}_{i}}^{(1)}+\sum_{j \in \Omega, j \neq i} h_{\mathrm{S}_{j} \mathrm{D}_{i}}^{(1)} x_{\mathrm{S}_{j}}^{(1)}+h_{\mathrm{CD}_{i}}^{(1)} x_{\mathrm{C}}^{(1)}+n_{\mathrm{D}_{i}}^{(1)}
$$

and the received signal at $\mathrm{D}_{i}$ through the relay link along with the direct one in timeslot 2 is written as

$$
y_{\mathrm{D}_{i}}^{(2)}=h_{\mathrm{S}_{i} \mathrm{D}_{i}}^{(2)} x_{\mathrm{S}_{i}}^{(2)}+\sum_{j \in \Omega, j \neq i} h_{\mathrm{S}_{j} \mathrm{D}_{i}}^{(2)} x_{\mathrm{S}_{j}}^{(2)}+h_{\mathrm{CD}_{i}}^{(2)} x_{\mathrm{C}}^{(2)}+n_{\mathrm{D}_{i}}^{(2)}+\beta h_{\mathrm{RD}_{i}}^{(2)}\left(h_{\mathrm{S}_{i} \mathrm{R}}^{(1)} x_{\mathrm{S}_{i}}^{(1)}+\sum_{j \in \Omega, j \neq i} h_{\mathrm{S}_{j} \mathrm{R}}^{(1)} x_{\mathrm{S}_{j}}^{(1)}+h_{\mathrm{CR}}^{(1)} x_{\mathrm{C}}^{(1)}+n_{\mathrm{R}}^{(1)}\right)
$$

where $h_{\mathrm{AB}}^{(t)}$ denote the channel gains between user $\mathrm{A}\left(\mathrm{A} \in\left\{\mathrm{S}_{i}, \mathrm{~S}_{j}, \mathrm{R}, \mathrm{C}\right\}\right)$ and user B ( $\left.\mathrm{B} \in\left\{\mathrm{D}_{i}, \mathrm{R}\right\}\right)$ in time-slot $t(t \in\{1,2\})$, which are assumed to be known at each user involved in the network by full-cooperation [21] and modeled as $h_{\mathrm{AB}}^{(t)}=c_{\mathrm{AB}}^{(t)} \sqrt{\left(d_{\mathrm{AB}}\right)^{-\alpha}}$, $d_{\mathrm{AB}}$ are the distances of $\mathrm{A} \rightarrow \mathrm{B}$ links, $c_{\mathrm{AB}}^{(t)}$ are the channel fading coefficients of these links, and $\alpha$ is the path loss exponent. In addition, $x_{\mathrm{Z}}^{(t)}$ denote the transmitted signals from user $\mathrm{Z}\left(\mathrm{Z} \in\left\{\mathrm{S}_{i}, \mathrm{~S}_{j}, \mathrm{C}\right\}\right), n_{\mathrm{B}}^{(t)}$ denote the additive noises at user B which follow the independent complex Gaussian distribution with zero mean and variance $\sigma^{2}$, and $\beta$ is the AF amplification factor [14]

Then, we define $\mathbf{y}_{\mathrm{D}_{i}}=\left[y_{\mathrm{D}_{i}}^{(1)}, y_{\mathrm{D}_{i}}^{(2)}\right]^{\mathrm{T}}, \mathbf{x}_{\mathrm{S}_{i}}=\left[x_{\mathrm{S}_{i}}^{(1)}, x_{\mathrm{S}_{i}}^{(2)}\right]^{\mathrm{T}}=\mathbf{v}_{i} s_{i}$ and $\mathbf{x}_{\mathrm{C}}=\left[x_{\mathrm{C}}^{(1)}, x_{\mathrm{C}}^{(2)}\right]^{\mathrm{T}}$, where the data symbol $s_{i}$ transmitted by $\mathrm{D}_{i}$ satisfies the expectation $\mathrm{E}\left[\left|s_{i}\right|^{2}\right]=P_{\mathrm{S}_{i}}$ ( $P_{\mathrm{S}_{i}}$ is the transmit power of $\mathrm{D}_{i}$ ), the $2 \times 1$ precoding vector $\mathbf{v}_{i}$ of $\mathrm{D}_{i}$ meets the 
power constraint $\mathbf{v}_{i}^{\mathrm{H}} \mathbf{v}_{i}=1, x_{\mathrm{C}}^{(1)}$ and $x_{\mathrm{C}}^{(2)}$ are independent and the expectations satisfy $\mathrm{E}\left[\left|x_{\mathrm{C}}^{(1)}\right|^{2}\right]=\mathrm{E}\left[\left|x_{\mathrm{C}}^{(2)}\right|^{2}\right]=P_{\mathrm{C}}\left(P_{\mathrm{C}}\right.$ is the transmit power of the cellular user $)$. By the above definitions, the signals received by $\mathrm{D}_{i}$ can be expressed in vector form as

$$
\mathbf{y}_{\mathrm{D}_{i}}=\mathbf{H}_{\mathrm{S}_{i} \mathrm{D}_{i}} \mathbf{x}_{\mathrm{S}_{i}}+\sum_{j \in \Omega, j \neq i} \mathbf{H}_{\mathrm{S}_{j} \mathrm{D}_{i}} \mathbf{x}_{\mathrm{S}_{j}}+\mathbf{H}_{\mathrm{CD}_{i}} \mathbf{x}_{\mathrm{C}}+\mathbf{n}_{\mathrm{D}_{i}}
$$

$$
\text { where } \mathbf{H}_{\mathrm{ZD}_{i}}=\left[\begin{array}{cc}
h_{\mathrm{ZD}_{i}}^{(1)} & 0 \\
\beta h_{\mathrm{RD}_{i}}^{(2)} h_{\mathrm{ZR}^{(1)}}^{(1)} & h_{\mathrm{ZD}_{i}}^{(2)}
\end{array}\right] \text { and } \mathbf{n}_{\mathrm{D}_{i}}=\left[\begin{array}{c}
n_{\mathrm{D}_{i}}^{(1)} \\
n_{\mathrm{D}_{i}}^{(2)}+\beta h_{\mathrm{RD}_{i}}^{(2)} n_{\mathrm{R}}^{(1)}
\end{array}\right] \text {. }
$$

Further, we define the $2 \times 1$ vector $\mathbf{u}_{i}$ as the corresponding decoding vector of $\mathrm{D}_{i}$, and hence the decoded signal at $\mathrm{D}_{i}$ can be obtained via multiplying (3) by $\mathbf{u}_{i}^{\mathrm{H}}$, i.e.,

$$
\hat{s}_{i}=\mathbf{u}_{i}^{\mathrm{H}} \mathbf{H}_{\mathrm{S}_{i} \mathrm{D}_{i}} \mathbf{x}_{\mathrm{S}_{i}}+\mathbf{u}_{i}^{\mathrm{H}} \sum_{j \in \Omega, j \neq i} \mathbf{H}_{\mathrm{S}_{j} \mathrm{D}_{i}} \mathbf{x}_{\mathrm{S}_{j}}+\mathbf{u}_{i}^{\mathrm{H}} \mathbf{H}_{\mathrm{CD}_{i}} \mathbf{x}_{\mathrm{C}}+\mathbf{u}_{i}^{\mathrm{H}} \mathbf{n}_{\mathrm{D}_{i}}
$$

where the first term on the right side of this formula is the desired signal, the second term plus the third one are the interferences, and the fourth term is the noise. With (4), the average power of the desired signal can be derived as

$$
\begin{aligned}
\mathrm{E}\left(\mathbf{u}_{i}^{\mathrm{H}} \mathbf{H}_{\mathrm{S}_{i} \mathrm{D}_{i}} \mathbf{x}_{\mathrm{S}_{i}} \mathbf{x}_{\mathrm{S}_{i}}^{\mathrm{H}} \mathbf{H}_{\mathrm{S}_{i} \mathrm{D}_{i}}^{\mathrm{H}} \mathbf{u}_{i}\right) & =\mathbf{u}_{i}^{\mathrm{H}} \mathbf{H}_{\mathrm{S}_{i} \mathrm{D}_{i}} \mathbf{v}_{i} \mathrm{E}\left(\left|s_{i}\right|^{2}\right) \mathbf{v}_{i}^{\mathrm{H}} \mathbf{H}_{\mathrm{S}_{i} \mathrm{D}_{i}}^{\mathrm{H}} \mathbf{u}_{i} \\
& =P_{\mathrm{S}_{i}} \mathbf{u}_{i}^{\mathrm{H}} \mathbf{H}_{\mathrm{S}_{i} \mathrm{D}_{i}} \mathbf{v}_{i} \mathbf{v}_{i}^{\mathrm{H}} \mathbf{H}_{\mathrm{S}_{i} \mathrm{D}_{i}}^{\mathrm{H}} \mathbf{u}_{i}
\end{aligned}
$$

Similarly, the average power of the interferences and noise can also be derived, and hence the SINR of each D2D receiver can be represented as

$$
\operatorname{SINR}_{i}\left(\mathbf{v}_{1}, \cdots, \mathbf{v}_{M}, \mathbf{u}_{i}\right)=\frac{P_{\mathrm{S}_{i}} \mathbf{u}_{i}^{\mathrm{H}} \mathbf{H}_{\mathrm{S}_{i} \mathrm{D}_{i}} \mathbf{v}_{i} \mathbf{v}_{i}^{\mathrm{H}} \mathbf{H}_{\mathrm{S}_{i} \mathrm{D}_{i}}^{\mathrm{H}} \mathbf{u}_{i}}{\mathbf{u}_{i}^{\mathrm{H}}\left(\sum_{j \in \Omega, j \neq i} P_{\mathrm{S}_{j}} \mathbf{H}_{\mathrm{S}_{j} \mathrm{D}_{i}} \mathbf{v}_{j} \mathbf{v}_{j}^{\mathrm{H}} \mathbf{H}_{\mathrm{S}_{j} \mathrm{D}_{i}}^{\mathrm{H}}+P_{\mathrm{C}} \mathbf{H}_{\mathrm{CD}_{i}} \mathbf{H}_{\mathrm{CD}_{i}}^{\mathrm{H}}+\mathbf{N}_{i}\right) \mathbf{u}_{i}}
$$

where $\mathbf{N}_{i}=\left[\begin{array}{cc}\sigma^{2} & 0 \\ 0 & \sigma^{2}\left(1+\beta^{2}\left|h_{\mathrm{RD}_{i}}^{(2)}\right|^{2}\right)\end{array}\right]$.

Finally, when the channel gains and the transmit power of users are fixed, the optimization problem can be formulated as

$$
\begin{array}{cl}
\max & \operatorname{SINR}_{i}\left(\mathbf{v}_{1}, \cdots, \mathbf{v}_{M}, \mathbf{u}_{i}\right) \\
\text { s.t. } & \mathbf{v}_{k}^{\mathrm{H}} \mathbf{v}_{k}=1, \quad \forall k \in \Omega
\end{array}
$$

\section{The SINR Improvement Algorithm for D2D Communication}

Apparently, it is difficult to work out the optimization problem (7) directly because multiple optimized variables exist. Therefore, in this section, we will first simplify the object function of the optimization problem through mathematical derivation on the basis of the generalized Rayleigh quotient and Rayleigh-Ritz theorem [22]. Then, we will clarify the relationship between the optimized variables in accordance with the simplified 
results, by which the original optimization problem can be transformed to one of solving nonlinear equations. Finally, we will combine the idea of Simulated Annealing (SA) metaheuristic [23] with the relationship between variables to propose the D2D SINR improvement algorithm (i.e., DSIA) solving the nonlinear equations.

First, by the property of generalized Rayleigh quotient, the maximum value $\lambda_{\max }\left[\mathbf{M}_{i}\right]$ of the objective function $\operatorname{SINR}_{i}\left(\mathbf{v}_{1}, \cdots, \mathbf{v}_{M}, \mathbf{u}_{i}\right)$ in (7) can be acquired when

$$
\mathbf{u}_{i}=\mathbf{e}_{\max }\left[\mathbf{M}_{i}\right]
$$

where

$$
\mathbf{M}_{i}=P_{\mathrm{S}_{i}} \mathbf{K}_{i}^{-1} \mathbf{H}_{\mathrm{S}_{i} \mathrm{D}_{i}} \mathbf{v}_{i} \mathbf{v}_{i}^{\mathrm{H}} \mathbf{H}_{\mathrm{S}_{i} \mathrm{D}_{i}}^{\mathrm{H}}
$$

and

$\mathbf{K}_{i}=\sum_{j \in \Omega, j \neq i} P_{\mathrm{S}_{j}} \mathbf{H}_{\mathrm{S}_{j} \mathrm{D}_{i}} \mathbf{v}_{j} \mathbf{v}_{j}^{\mathrm{H}} \mathbf{H}_{\mathrm{S}_{j} \mathrm{D}_{i}}^{\mathrm{H}}+P_{\mathrm{C}} \mathbf{H}_{\mathrm{CD}_{i}} \mathbf{H}_{\mathrm{CD}_{i}}^{\mathrm{H}}+\mathbf{N}_{i}$. Since $\mathbf{K}_{i}$ is an invertible matrix, the rank of $\mathbf{M}_{i}$ will be

$$
\operatorname{rank}\left[\mathbf{M}_{i}\right]=\operatorname{rank}\left[\mathbf{H}_{\mathrm{S}_{i} \mathrm{D}_{i}} \mathbf{v}_{i} \mathbf{v}_{i}^{\mathrm{H}} \mathbf{H}_{\mathrm{S}_{i} \mathrm{D}_{i}}^{\mathrm{H}}\right]=1
$$

this means that in the two eigenvalues of $\mathbf{M}_{i}$, one is zero and the other is a non-zero value. As $\mathbf{K}_{i}^{-1}$ is positive definite ( $\mathbf{K}_{i}$ is positive definite), $\mathbf{H}_{\mathrm{S}_{i} \mathrm{D}_{i}} \mathbf{v}_{i} \mathbf{v}_{i}^{\mathrm{H}} \mathbf{H}_{\mathrm{S}_{i} \mathrm{D}_{i}}^{\mathrm{H}}$ is positive semi-definite, and they are both Hermite matrices, the eigenvalues of $\mathbf{M}_{i}$ must be greater than or equal to zero [24]. Hence, the non-zero eigenvalue will be greater than zero, and should be equal to $\lambda_{\max }\left[\mathbf{M}_{i}\right]$. Further, since the trace of $\mathbf{M}_{i}$ is the sum of 0 and $\lambda_{\max }\left[\mathbf{M}_{i}\right]$, substituting (8) into $\operatorname{SINR}_{i}\left(\mathbf{v}_{1}, \cdots, \mathbf{v}_{M}, \mathbf{u}_{i}\right)$ and problem (7) can be written as

$$
\begin{array}{cc}
\max & \operatorname{tr}\left[\mathbf{M}_{i}\left(\mathbf{v}_{1}, \cdots, \mathbf{v}_{M}\right)\right] \\
\text { s.t. } & \mathbf{v}_{k}^{\mathrm{H}} \mathbf{v}_{k}=1, \quad \forall k \in \Omega
\end{array}
$$

By using the property of trace, the objective function in (10) can be transformed to

$$
\begin{aligned}
\operatorname{tr}\left[\mathbf{M}_{i}\right] & =\operatorname{tr}\left[\mathbf{H}_{\mathrm{S}_{i} \mathrm{D}_{i}}^{\mathrm{H}} \mathbf{K}_{i}^{-1} \mathbf{H}_{\mathrm{S}_{i} \mathrm{D}_{i}} \mathbf{v}_{i} \mathbf{v}_{i}^{\mathrm{H}}\right] \\
& =\mathbf{v}_{i}^{\mathrm{H}} \mathbf{H}_{\mathrm{S}_{i} \mathrm{D}_{i}}^{\mathrm{H}} \mathbf{K}_{i}^{-1} \mathbf{H}_{\mathrm{S}_{i} \mathrm{D}_{i}} \mathbf{v}_{i}
\end{aligned}
$$

and hence problem (10) can be rewritten as

$$
\begin{aligned}
& \max \left\{\begin{array}{l}
\mathbf{v}_{i}^{\mathrm{H}} \mathbf{H}_{\mathrm{S}_{\mathrm{i}} \mathrm{D}_{i}}^{\mathrm{H}}\left[\mathbf{K}_{i}\left(\mathbf{v}_{2}, \cdots, \mathbf{v}_{M}\right)\right]^{-1} \mathbf{H}_{\mathrm{S}_{i} \mathrm{D}_{i}} \mathbf{v}_{i} \quad \text { if } i=1, \\
\mathbf{v}_{i}^{\mathrm{H}} \mathbf{H}_{\mathrm{S}_{i} \mathrm{D}_{i}}^{\mathrm{H}}\left[\mathbf{K}_{i}\left(\mathbf{v}_{1} \cdots \mathbf{v}_{i-1}, \mathbf{v}_{i+1} \cdots \mathbf{v}_{M}\right)\right]^{-1} \mathbf{H}_{\mathrm{S}_{i} \mathrm{D}_{i}} \mathbf{v}_{i} \quad \text { if } 1<i<M, \\
\mathbf{v}_{i}^{\mathrm{H}} \mathbf{H}_{\mathrm{S}_{i} \mathrm{D}_{i}}^{\mathrm{H}}\left[\mathbf{K}_{i}\left(\mathbf{v}_{1}, \cdots, \mathbf{v}_{M-1}\right)\right]^{-1} \mathbf{H}_{\mathrm{S}_{i} \mathrm{D}_{i}} \mathbf{v}_{i} \quad \text { if } i=M .
\end{array}\right. \\
& \text { s.t. } \quad \mathbf{v}_{k}^{\mathrm{H}} \mathbf{v}_{k}=1, \quad \forall k \in \Omega
\end{aligned}
$$

With the use of the Rayleigh-Ritz theorem, the maximum value $\lambda_{\max }\left[\mathbf{Q}_{i}\right]$ of the objective function in (12) can be acquired when

where $\mathbf{Q}_{i}=\mathbf{H}_{\mathrm{S}_{i} \mathrm{D}_{i}}^{\mathrm{H}} \mathbf{K}_{i}^{-1} \mathbf{H}_{\mathrm{S}_{i} \mathrm{D}_{i}}$.

$$
\mathbf{v}_{i}=\mathbf{e}_{\max }\left[\mathbf{Q}_{i}\right]
$$




\section{Algorithm: DSIA}

Defining the initial temperature as $T_{0}$, the annealing control parameter as $r$, the lower limit of temperature as $T_{\min }$, and the amount of inner loop as $\delta$. Initializing the precoding vector of each D2D transmitter as arbitrary $\mathbf{v}_{k}^{(0)}(\forall k \in \Omega)$ with the power constraint $\left[\mathbf{v}_{k}^{(0)}\right]^{\mathrm{H}} \mathbf{v}_{k}^{(0)}=1$, and temp $=T_{0}$.

while temp $>T_{\min }$

for $l=1$ to $\delta$

Calculating the precoding vector of each D2D transmitter:

$$
\left\{\begin{array}{l}
\mathbf{v}_{i}^{(l)}=\mathbf{e}_{\max }\left[\mathbf{Q}_{i}\left(\mathbf{v}_{2}^{(l-1)}, \cdots, \mathbf{v}_{M}^{(l-1)}\right)\right] \quad \text { if } i=1, \\
\mathbf{v}_{i}^{(l)}=\mathbf{e}_{\max }\left[\mathbf{Q}_{i}\left(\mathbf{v}_{1}^{(l-1)} \cdots \mathbf{v}_{i-1}^{(l-1)}, \mathbf{v}_{i+1}^{(l-1)} \cdots \mathbf{v}_{M}^{(l-1)}\right)\right] \quad \text { if } 1<i<M, \\
\mathbf{v}_{i}^{(l)}=\mathbf{e}_{\max }\left[\mathbf{Q}_{i}\left(\mathbf{v}_{1}^{(l-1)}, \cdots, \mathbf{v}_{M-1}^{(l-1)}\right)\right] \quad \text { if } i=M .
\end{array}\right.
$$

Calculating the SINR increment at each D2D receiver:

$$
\left\{\begin{array}{l}
\Delta_{i}=\lambda_{\max }\left[\mathbf{Q}_{i}\left(\mathbf{v}_{2}^{(l)}, \cdots, \mathbf{v}_{M}^{(l)}\right)\right]-\lambda_{\max }\left[\mathbf{Q}_{i}\left(\mathbf{v}_{2}^{(l-1)}, \cdots, \mathbf{v}_{M}^{(l-1)}\right)\right] \quad \text { if } i=1, \\
\Delta_{i}=\lambda_{\max }\left[\mathbf{Q}_{i}\left(\mathbf{v}_{1}^{(l)} \cdots \mathbf{v}_{i-1}^{(l)}, \mathbf{v}_{i+1}^{(l)} \cdots \mathbf{v}_{M}^{(l)}\right)\right]-\lambda_{\max }\left[\mathbf{Q}_{i}\left(\mathbf{v}_{1}^{(l-1)} \cdots \mathbf{v}_{i-1}^{(l-1)}, \mathbf{v}_{i+1}^{(l-1)} \cdots \mathbf{v}_{M}^{(l-1)}\right)\right] \quad \text { if } 1<i<M \\
\Delta_{i}=\lambda_{\max }\left[\mathbf{Q}_{i}\left(\mathbf{v}_{1}^{(l)}, \cdots, \mathbf{v}_{M-1}^{(l)}\right)\right]-\lambda_{\max }\left[\mathbf{Q}_{i}\left(\mathbf{v}_{1}^{(l-1)}, \cdots, \mathbf{v}_{M-1}^{(l-1)}\right)\right] \quad \text { if } i=M .
\end{array}\right.
$$

if $\Delta_{i} \geq 0$

Updating $\mathbf{v}_{i}^{(l)} \leftarrow \mathbf{v}_{i}^{(l-1)}$

else

Updating $\mathbf{v}_{i}^{(l)} \leftarrow \mathbf{v}_{i}^{(l-1)}$ with the probability $\exp \left(\frac{\Delta_{i}}{\text { temp }}\right)$

end if

$l=l+1$

end for

Resetting $\mathbf{v}_{i}^{(0)}=\mathbf{v}_{i}^{(\delta)}$

Annealing as temp $=r \cdot$ temp

end while

Outputting the updated precoding vectors as the optimized results: $\left\{\mathbf{v}_{1}^{\text {opt }}, \cdots, \mathbf{v}_{M}^{\text {opt }}\right\}$.

Based on the optimized precoding vectors, calculating the decoding vector of each D2D receiver: $\mathbf{u}_{i}=\mathbf{e}_{\max }\left[\mathbf{M}_{i}\left(\mathbf{v}_{1}^{\mathrm{opt}}, \cdots, \mathbf{v}_{M}^{\mathrm{opt}}\right)\right]$.

Figure 2. The D2D SINR Improvement Algorithm: DSIA

Then, with the derivation in (8)-(13), it demonstrates that the optimized variables are correlated with each other. More precisely, in the optimization process, $\mathbf{u}_{i}$ is determined by $\left\{\mathbf{v}_{1}, \cdots, \mathbf{v}_{M}\right\}$, while $\mathbf{v}_{i}$ is determined by all $\mathbf{v}_{j}(j \in \Omega, j \neq i)$. For a certain D2D pair, this means that the decoding vector of its receiver can only be 
determinate when the precoding vectors of all D2D transmitters are determinate, and the precoding vector of its transmitter are intertwined with that of other D2D transmitters.

Finally, through the above analysis, it clearly indicates that the next work is to solve the following nonlinear equations:

$$
\left\{\begin{array}{c}
\mathbf{v}_{1}=\mathbf{e}_{\max }\left[\mathbf{Q}_{1}\right] \\
\vdots \\
\mathbf{v}_{M}=\mathbf{e}_{\max }\left[\mathbf{Q}_{M}\right]
\end{array}\right.
$$

Obviously, it does not guarantee that the function in (14) is continuous, and hence some traditional iterative methods to solve nonlinear equations (such as Newton, Chebyshev, and Halley methods, etc.) are not applicable. Therefore, the Simulated Annealing (SA) metaheuristic is taken into consideration. SA has strong robustness and global convergence, furthermore, it has theoretically been proved to be an optimization method converges to a global optimal point or an approximate global optimal point with probability one [25]. Based on the idea of SA, we combined with the relationship between variables to propose a D2D SINR improvement algorithm called DSIA solving (14), which is shown in Figure 2 at the top of the previous page.

In conclusion, the DSIA treats all D2D pairs in the network equally, and guarantees all D2D receivers' SINR being maximized based on the optimized precoding and decoding vectors obtained by it. In addition, the DSIA mainly focus on the scenario where multiple D2D pairs $(M \geq 2)$ exist, if there is only one D2D pair $(M=1)$ in the network, there is no need to adopt this algorithm, and we can directly obtain closed-form solutions of optimized vectors and the upper boundary of D2D receivers' SINR in this scenario by the analysis from (8)-(13), where it can be understood as the other D2D transmitters are turned off or their corresponding precoding vectors adopt the zero vector.

\section{Numerical Results}

In this section, we present several simulations and numerical results to verify the performance of the proposed algorithm. In general, channel fading coefficients of the links existing in the network are assumed to be independent identically distributed complex Gaussian variables with zero mean and unit variance. Besides, the number of D2D pairs is set as $M=2$, and in order to keep the total transmit powers constant and make a fair comparison with the spectrum orthogonal scheme, we assume that the sum of $P_{\mathrm{R}}$ (the transmit power of the introduced relay) and $\left(P_{\mathrm{S}_{1}}+P_{\mathrm{S}_{2}}\right)$ (the total transmit power of all D2D transmitters in this paper $)$ is equal to $P_{\mathrm{S}}\left(P_{\mathrm{S}}=\tilde{P}_{\mathrm{S}_{1}}+\tilde{P}_{\mathrm{S}_{2}}\right.$, and it denotes the total transmit power of all D2D transmitters in the scenario without the introducing of the relay). For the sake of simplicity and clarity, we assume that $P_{\mathrm{S}_{1}}=P_{\mathrm{S}_{2}}, \tilde{P}_{\mathrm{S}_{1}}=\tilde{P}_{\mathrm{S}_{2}}$, and call $P_{\mathrm{S}}$ as the D2D transmit power. Furthermore, $T_{0}=1, T_{\min }=10^{-5}, r=0.8$ and $\delta=10$ are set in the DSIA according to [25]. Other key simulation parameters are given as follows. The path loss exponent is 2 , the cell radius is $500 \mathrm{~m}$, the communication distances for both D2D pairs are $10 \mathrm{~m}$, the distances between the cellular user and both D2D receivers are $50 \mathrm{~m}$, the distances between the relay and both D2D transceivers are $10 \mathrm{~m}$, and the initialized precoding vector of each D2D transmitter is $[\sqrt{2} / 2, \sqrt{2} / 2]$.

Figure 3 shows the SINR of both D2D receivers with different proportions of the relay power in the D2D transmit power (i.e., $P_{\mathrm{R}} / P_{\mathrm{S}}$ ), where $P_{\mathrm{S}}=P_{\mathrm{C}}$ and $P_{\mathrm{S}} / \sigma^{2}=20 \mathrm{~dB}$. From this figure, we can see that by using the DSIA, the SINR of both D2D receivers are highly 
consistent and being significantly improved. For example, when $P_{\mathrm{R}} / P_{\mathrm{S}}=3 / 10$, the DSIA enables D2D to achieve a SINR gain of about $17.81 \%$ over that using the spectrum orthogonal scheme, and achieve a SINR gain of about $21.59 \%$ over the case with no interference control. Besides, this figure shows that the SINR performance of D2D using the DSIA will degrade with the increase of $P_{\mathrm{R}} / P_{\mathrm{S}}$, this is because the increasing relay power will amplify the power of the retransmitted interference and noise, which leads to the performance degradation.

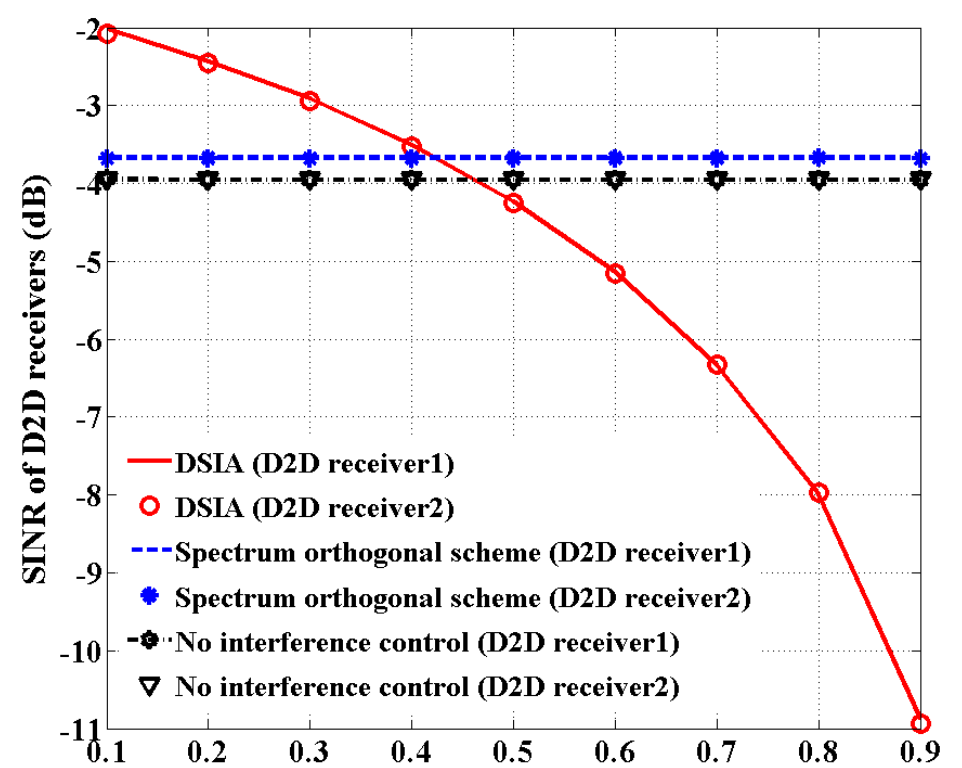

The proportion of the relay power in the D2D transmit power: $P_{R} / P_{S}$

\section{Figure 3. SINR of D2D Receivers with Different Proportions of the Relay Power in the D2D Transmit Power}

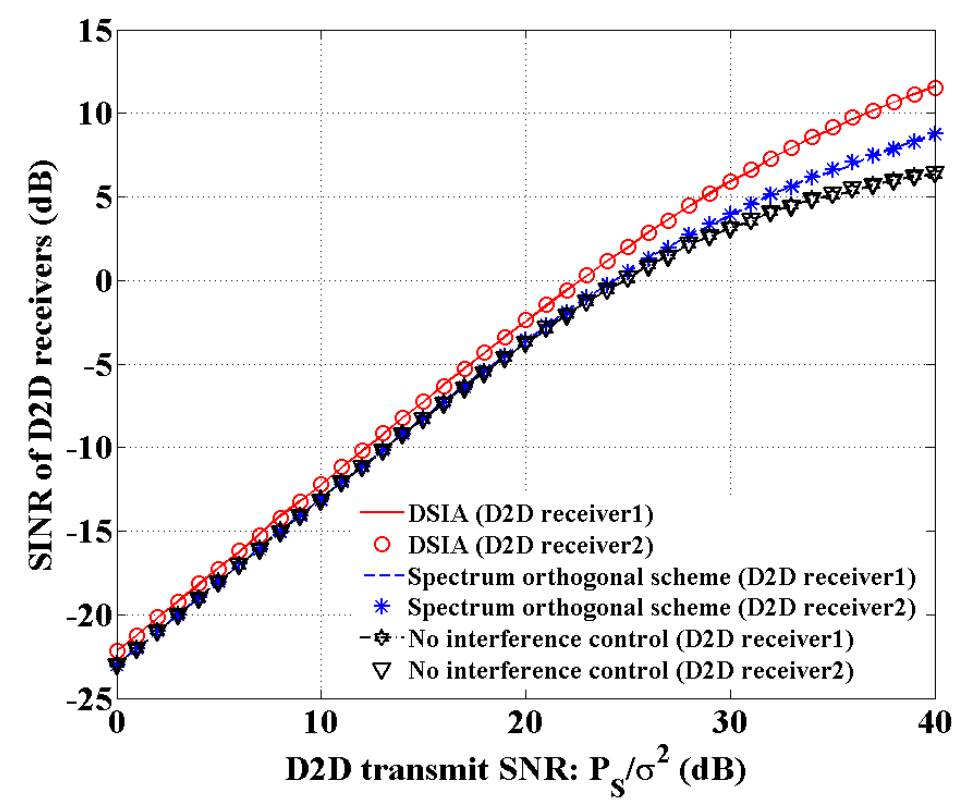

Figure 4. SINR of D2D Receivers with Different D2D Transmit SNR

Based on the analysis about Figure 3 with the fixed D2D transmit SNR (i.e., $P_{\mathrm{S}} / \sigma^{2}$ ), Figure 4 and Figure 5 further show the SINR and BER of both D2D receivers with 
different values of $P_{\mathrm{S}} / \sigma^{2}$, where $P_{\mathrm{S}}=P_{\mathrm{C}}$ and $P_{\mathrm{R}} / P_{\mathrm{S}}=1 / 5$. From the two figures, we can also see that the DSIA will enable D2D to obtain obvious performance gains in terms of SINR and BER. For example, when $P_{\mathrm{S}} / \sigma^{2}=20 \mathrm{~dB}$, D2D using the DSIA can achieve a SINR gain of $27.64 \%$ and a BER gain of $23.11 \%$ over that using the spectrum orthogonal scheme, while compared with the case with no interference control, the SINR and BER gains are more significant, which reach $32.01 \%$ and $25.74 \%$ respectively. Obviously, the above SINR gains are influenced by the value of $P_{\mathrm{R}} / P_{\mathrm{S}}$, which proof the correctness of analysis about Figure 3.

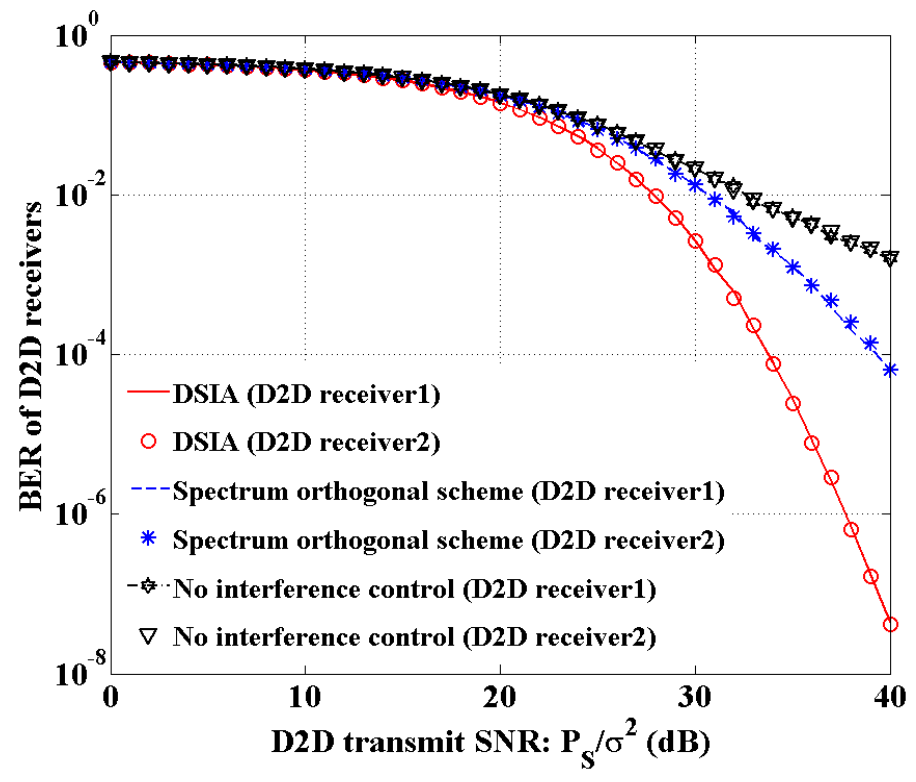

Figure 5. BER of D2D Receivers with Different D2D Transmit SNR

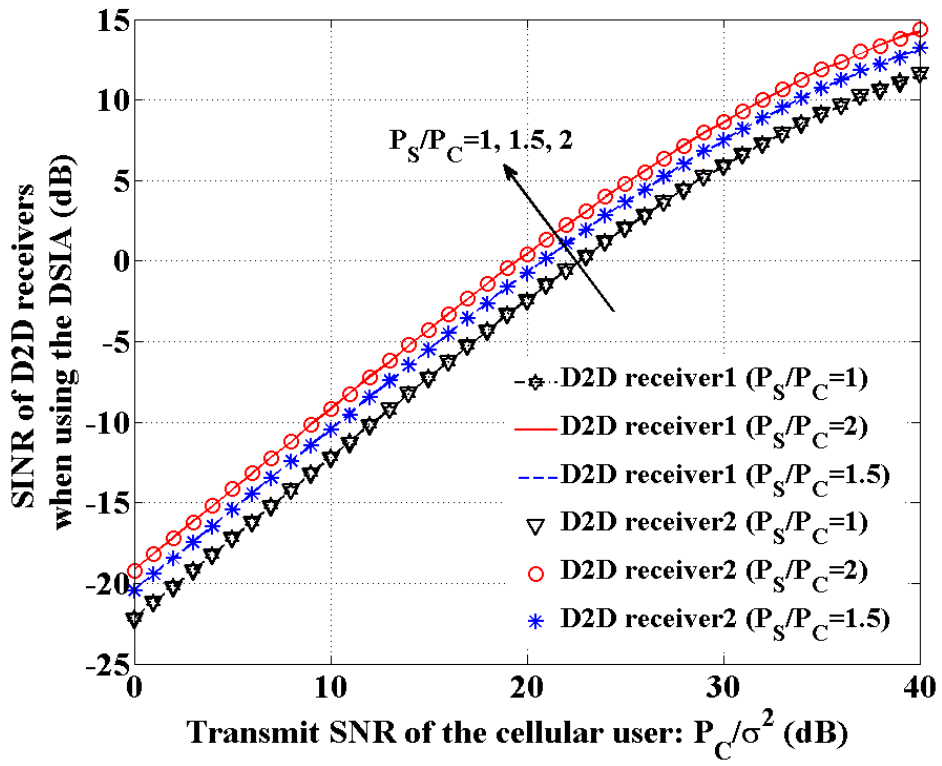

Figure 6. SINR of D2D Receivers with Different Transmit SNR of the Cellular User

In Figure 3-5, we have presented the SINR and BER performances of D2D receivers when using the DSIA, under the assumption that the D2D transmit power and the transmit 
power of the cellular user are equal (i.e., $P_{\mathrm{S}}=P_{\mathrm{C}}$ ), which refers to the strong interference regime [5]. In Figure 6 and Figure 7, we will investigate the two performances in the weak interference regime [5], and accordingly the values of $P_{\mathrm{S}} / P_{\mathrm{C}}$ are set as 1.5 and 2, respectively. Note that for comparison purposes, the case when $P_{\mathrm{S}} / P_{\mathrm{C}}=1$ is also shown in the following two figures.

Figure 6 and Figure 7 show the SINR and BER of both D2D receivers with different transmit SNR of the cellular user (i.e., $P_{\mathrm{C}} / \sigma^{2}$ ), where $P_{\mathrm{R}} / P_{\mathrm{S}}=1 / 5$. From the two figures, we can see that the increase of $P_{\mathrm{S}} / P_{\mathrm{C}}$ will enable D2D using the DSIA to achieve better performance gains in terms of SINR and BER. For example, when $P_{\mathrm{C}} / \sigma^{2}=20 \mathrm{db}$, the one adopts $P_{\mathrm{S}} / P_{\mathrm{C}}=1.5$ achieves a SINR gain of $47.44 \%$ and a BER gain of $49.42 \%$ over the one adopts $P_{\mathrm{S}} / P_{\mathrm{C}}=1$, while the one adopts $P_{\mathrm{S}} / P_{\mathrm{C}}=2$ achieves a SINR gain of $23.75 \%$ and a BER gain of $44.21 \%$ over the one adopts $P_{\mathrm{S}} / P_{\mathrm{C}}=1.5$.

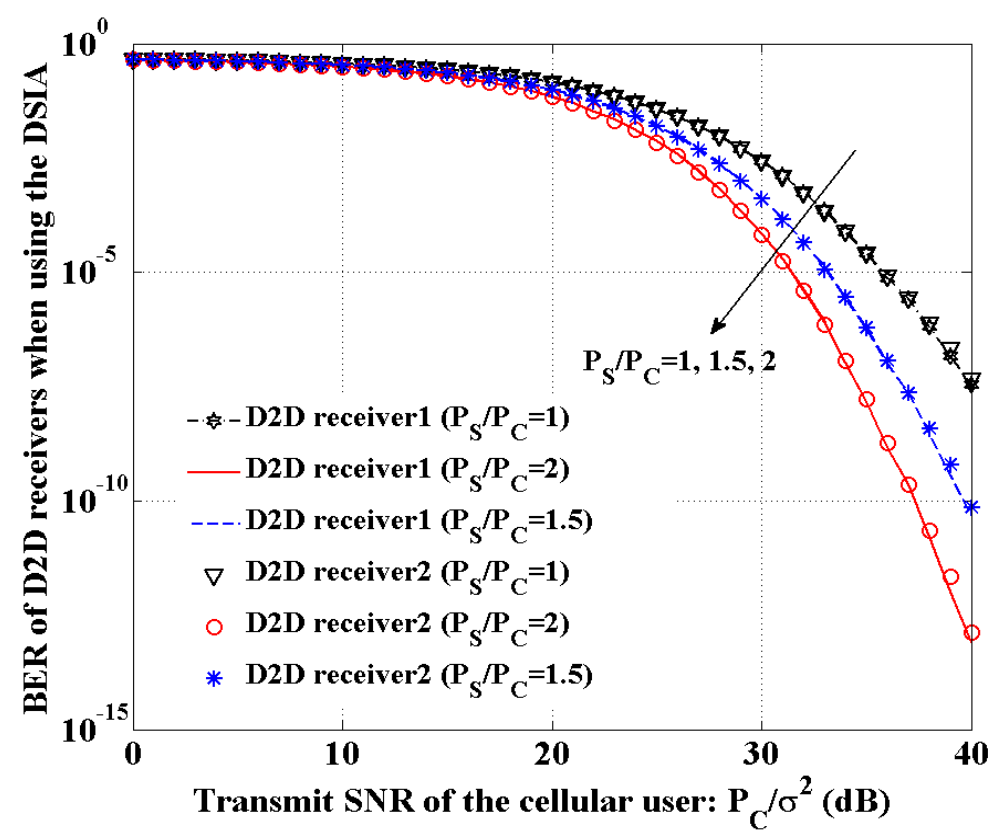

Figure 7. BER of D2D Receivers with Different Transmit SNR of the Cellular User

\section{Conclusion}

In this paper, we propose a novel D2D SINR improvement algorithm (DSIA) in the interference scenario where multiple D2D pairs and one cellular user coexist and they share the cellular uplink resources. The multi-variable optimization problem to maximize the SINR of each D2D receiver is first formulated according to the D2D system with the introducing of a green AF relay, then the original optimization problem is transformed to one of solving nonlinear equations by mathematical derivation, and finally the DSIA is proposed to work out the nonlinear equations. Numerical results show that D2D communication using the DSIA will make all D2D receivers obtain the same SINR and BER performances, while compared with that using the traditional spectrum orthogonal scheme and the case with no interference control, significant performance gains in terms of SINR and BER can be achieved if the certain relay power is adopted, as well as the D2D transmit power increase. The proposed method suggests a promising direction to improve the system performance of D2D communication, and it can be applied to the 
more complicated interference scenario where multiple D2D pairs and cellular users share the same spectrum resources in future research.

\section{Acknowledgments}

This paper is a revised and expanded version of a paper entitled "A SINR Improvement Algorithm for D2D Communication Underlaying Cellular Networks" presented at CST 2016, Suzhou, China, April 22-23, 2016. This work was supported by the Natural Science Foundation of China (61372126, 61302101, 61402234), the Natural Science Foundation of Jiangsu Province of China (20130874), the Open Research Fund of National Mobile Communications Research Laboratory in Southeast University (Grant No. 2012D11) and the Graduate Innovation Plan of Jiangsu Province (CXZZ13_0483). Dr. Cheng Huan is the corresponding author.

\section{References}

[1] G. Fodor, E. Dahlman, G. Mildh, S. Parkvall, N. Reider, G. Miklos and Z. Turanyi, "Design Aspects of Network Assisted Device-to-Device Communications", IEEE Communications Magazine, vol. 50, no. 3, (2012), pp. 170-177.

[2] K. Doppler, M. Rinne, C. Wijting, C. Ribeiro and K. Hug, "Device-to-Device Communication as an Underlay to LTE-Advanced Networks", IEEE Communications Magazine, vol. 47, no. 12, (2009), pp. 42-49.

[3] D. Feng, L. Lu, Y. Yi, G. Y. Li, G. Feng and S. Li, "Device-to-Device Communications Underlaying Cellular Networks", IEEE Transactions on Communications, vol. 61, no. 8, (2013), pp. 3541-3551.

[4] Y. C. Chen, S. B. He, F. Hou, Z. G. Shi and X. Chen, "Optimal User-Centric Relay Assisted Device-toDevice Communications: an Auction Approach", IET Communications, vol. 9, no. 3, (2015), pp. 386395.

[5] H. Min, W. Seo, J. Lee, S. Park and D. Hong, "Reliability Improvement Using Receive Mode Selection in the Device-to-Device Uplink Period Underlaying Cellular Networks", IEEE Transactions on Wireless Communications, vol. 10, no. 2, (2011), pp. 413-418.

[6] H. Min, J. Lee, S. Park and D. Hong, "Capacity Enhancement Using an Interference Limited Area for Device-to-Device Uplink Underlaying Cellular Networks", IEEE Transactions on Wireless Communications, vol. 10, no. 12, (2011), pp. 3995-4000.

[7] C. H. Yu, O. Tirkkonen, K. Doppler and C. Ribeiro, "On the Performance of Device-to-Device Underlay Communication with Simple Power Control", Proceedings of IEEE 69th Vehicular Technology Conference (VTC), Barcelona, Spain, (2009).

[8] D. Zhu, W. Xu, H. Zhang, C. Zhao, J. C. F. Li and M. Lei, "Rate-Maximized Transceiver Optimization for Multi-Antenna Device-to-Device Communications", Proceedings of IEEE Wireless Communications and Networking Conference (WCNC), Shanghai, China, (2013).

[9] P. Janis, V. Koivunen, C. B. Ribeiro, K. Doppler and K. Hugl, "Interference-Avoiding MIMO Schemes for Device-to-Device Radio Underlaying Cellular Networks", Proceedings of IEEE 20th Personal, Indoor and Mobile Radio Communications Symposium (PIMRC), Tokyo, Japan, (2009).

[10] W. Xu, L. Liang, H. Zhang, S. Jin, J. C. F. Li and M. Lei, "Performance Enhanced Transmission in Device-to-Device Communications: Beamforming or Interference Cancellation", Proceedings of IEEE Global Communications Conference (GLOBECOM), Anaheim, CA, United states, (2012).

[11] H. E. Elkotby, K. M. F. Elsayed and M. H. Ismail, "Exploiting Interference Alignment for Sum Rate Enhancement in D2D-Enabled Cellular Networks", Proceedings of IEEE Wireless Communications and Networking Conference (WCNC), Paris, France, (2012).

[12] V. R. Cadambe and S. A. Jafar, "Interference Alignment and the Degrees of Freedom of the K User Interference Channel", IEEE Transactions on Information Theory, vol. 54, no. 8, (2008), pp. 3425-3441.

[13] V. R. Cadambe, S. A. Jafar, H. Maleki, K. Ramchandran and C. Suh, "Asymptotic Interference Alignment for Optimal Repair of MDS Codes in Distributed Storage", IEEE Transactions on Information Theory, vol. 59, no. 5, (2013), pp. 2974-2987.

[14] J. Laneman, D. N. C. Tse and G. W. Wornell, "Cooperative Diversity in Wireless Networks: Efficient Protocols and Outage Behavior", IEEE Transactions on Information Theory, vol. 50, no. 12, (2004), pp. 3062-3080.

[15] H. Ning, C. Ling and K. K. Leung, "Relay-Aided Interference Alignment: Feasibility Conditions and Algorithm", Proceedings of IEEE International Symposium on Information Theory (ISIT), Austin, TX, United states, (2010).

[16] K. Gomadam, V. R. Cadambe and S. A. Jafar, "A Distributed Numerical Approach to Interference Alignment and Applications to Wireless Interference Networks", IEEE Transactions on Information Theory, vol. 57, no. 6, (2011), pp. 3309-3322. 
[17] K. Gomadam, V. R. Cadambe and S. A. Jafar, "Approaching the Capacity of Wireless Networks through Distributed Interference Alignment", Proceedings of IEEE Global Telecommunications Conference (GLOBECOM), New Orleans, LA, United states, (2008).

[18] T. Han and N. Ansari, "Heuristic Relay Assignments for Green Relay Assisted Device-to-Device Communications", Proceedings of IEEE Global Communications Conference (GLOBECOM), Atlanta, GA, United states, (2013).

[19] M. H. Xia and S. Aissa, "Spectrum-Sharing Multi-Hop Cooperative Relaying: Performance Analysis Using Extreme Value Theory", IEEE Transactions on Wireless Communications, vol. 13, no. 1, (2014), pp. 234-245.

[20] C. Huan, Y. H. Fu and J. Wang, "A SINR Improvement Algorithm for D2D Communication Underlaying Cellular Networks", Proceedings of Advanced Science and Technology Letters (ASTL): the 5th International Conference on Computer Science and Technology (CST), Suzhou, China, (2016).

[21] Z. Liu, T. Peng, B. Peng and W. Wang, "Sum-Capacity of D2D and Cellular Hybrid Networks over Cooperation and Non-Cooperation", Proceedings of 7th International ICST Conference on Communications and Networking in China (CHINACOM), Kun Ming, China, (2012).

[22] X. Zhang, "Matrix Analysis and Applications", Tsinghua University Press, Beijing, (2004).

[23] F. Glover and G. A. Kochenberger, "Handbook of Metaheuristics", Kluwer, New York, (2003).

[24] C. L. Zhu, "The Eigenvalue of the Product of Positive Semi-Definite Matrix", Journal of Huaiyin Teachers College, vol. 2, no.3, (2003), pp. 186-189.

[25] S. Bandyopadhyay, S. Saha, U. Maulik and K. Deb, "A Simulated Annealing-Based Multiobjective Optimization Algorithm: AMOSA", IEEE Transactions on Evolutionary Computation, vol. 12, no. 3, (2008), pp. 269-283.
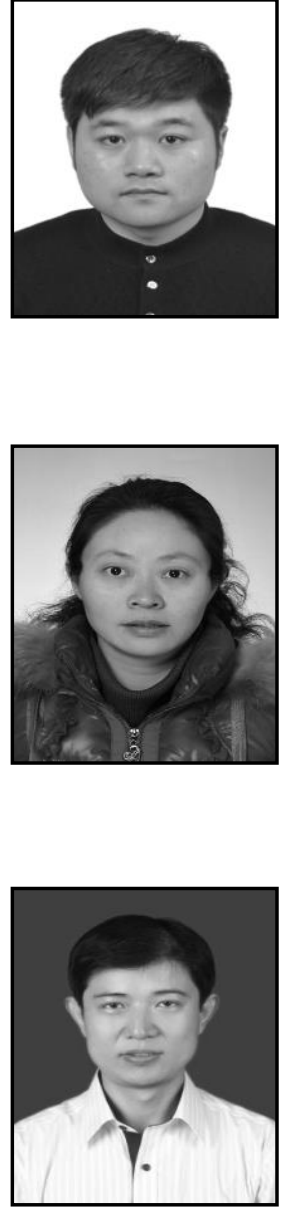

\section{Authors}

Cheng Huan, he received the B.S. degree in optoelectronic engineering from Nanjing University of Posts and Telecommunications, China, in 2008 and the M.S. degree in circuits and systems from Chongqing University of Posts and Telecommunications, China, in 2011. Currently, he is pursuing the $\mathrm{Ph} . \mathrm{D}$. degree in communication and information systems from Nanjing University of Posts and Telecommunications, China. His current research interests include D2D communication and cooperative communication.

Youhua Fu, she received the B.S. degree in communication engineering and the M.S. degree in communication and information systems from the School of Information Science and Technology, Southwest Jiaotong University, Chengdu, China, in 2000 and 2003, respectively. She received the Ph.D. degree in signal and information processing from the School of Information Science and Engineering, Southeast University, Nanjing, China, in 2010. Her current research interests include MIMO communication systems and cooperative communication.

Jin Wang, he received the B.S. and M.S. degree from Nanjing University of Posts and Telecommunications, China, in 2002 and 2005, respectively. He received Ph.D. degree from Kyung Hee University, Korea, in 2010. Now, he is a professor in the Computer and Software Institute, Nanjing University of Information Science and technology. His research interests mainly include routing method and algorithm design, performance evaluation and optimization for wireless ad hoc and sensor networks. He is a member of the IEEE and ACM. 